\title{
Réflexions sur un nouveau statut de la Guyane
}

\section{Thierry Michalon}

\section{(2) OpenEdition \\ Journals}

Édition électronique

URL : http://journals.openedition.org/plc/528

DOI : $10.4000 /$ plc.528

ISSN : 2117-5209

\section{Éditeur}

L'Harmattan

Édition imprimée

Date de publication : 1 janvier 1999

Pagination : 253-279

ISSN : 1279-8657

\section{Référence électronique}

Thierry Michalon, «Réflexions sur un nouveau statut de la Guyane », Pouvoirs dans la Caraïbe [En ligne] 11 | 1999, mis en ligne le 08 mars 2011, consulté le 01 mai 2019. URL : http:// journals.openedition.org/plc/528 ; DOI : 10.4000/plc.528 


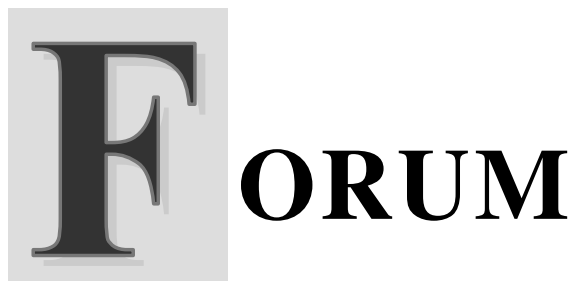

\section{Réflexions sur un nouveau statut de la Guyane}

Depuis quelques années mûrit en silence une réflexion autour d'une réforme du statut de la Guyane. Plusieurs raisons conduisent à y accorder un intérêt particulier.

En premier lieu, le Conseil général de Guyane, soucieux d'éviter une multiplication des institutions dans un territoire peu peuplé, a, seul parmi les Conseils généraux d'outre-mer, rendu le 22 juillet 1982 un avis favorable au projet de loi instaurant un département et une région dotés d'organes communs; on le sait, cette loi, adoptée le 23 novembre suivant, fut déclarée non conforme à la Constitution par le Conseil constitutionnel dans sa décision du 2 décembre, et le législateur dut dans la hâte se résoudre à en revenir à des organes distincts. Depuis lors les élus guyanais prennent régulièrement position contre cette peu défendable superposition institutionnelle, qu'un rapport Suchod pour la Commission des lois de l'Assemblée nationale avait d'ailleurs, à l'époque, sévèrement critiquée. Les présidents successifs des assemblées départementale et régionale ont, à Cayenne, continûment dénoncé les coûts inutilement élevés, les rivalités, les difficultés dans la répartition des compétences, la mauvaise coordination, notamment, qu'entraîne cette dualité 
institutionnelle. Aussi la demande d'un retour à une "assemblée unique » assortit-elle avec constance celle d'une décentralisation plus poussée, voire d'une autonomie pour la Guyane.

En second lieu, le Secrétariat d'Etat à l'Outre-mer semble prêter aux demandes de réforme statutaire venues de Cayenne une oreille plus attentive qu'il ne le fait pour les trois départements d'outre-mer insulaires, et s'en explique sans réticence. Alors que la Guadeloupe, la Martinique et la Réunion s'enfoncent chaque jour un peu plus dans les impasses socio-économiques où la logique départementale, associée à la simple démographie, les conduit depuis plusieurs décennies, la Guyane, pour sa part, jouirait encore d'une réelle marge de manœuvre :

- avec environ 150000 habitants, ce territoire de $91000 \mathrm{~km}^{2}$ (plus du double de la superficie de la Suisse) demeure très peu peuplé, et peu mis en valeur ;

- les différentes administrations (Etat, région, département) offrent à cette population, numériquement très inférieure à celle des D.O.M. insulaires (390 000 habitants pour la Martinique, 400000 pour la Guadeloupe, 660000 pour la Réunion) un volume d'emplois publics du même ordre ;

- le Centre spatial guyanais emploie environ 700 Guyanais et finance, à travers le plan « P.H.E.D.R.E. », des travaux d'infrastructures générateurs d'emplois ;

- une partie de la population a conservé son mode de vie et ses activités productives traditionnelles, demeure donc en partie indemne des sentiments de dépendance économique et d'aliénation culturelle qui suscitent, dans les trois autres départements d'outre-mer, tant de frustrations, et ne manifeste pas, comme dans les trois îles, d'exigences de mode de vie incompatibles avec le niveau de productivité de l'économie locale ;

- les activités de pêche industrielle s'avèrent prospères et susceptibles d'expansion ; 
- l'exploitation du bois, malgré divers facteurs limitants, s'est développée.

En d'autres termes, la Guyane présenterait des perspectives, encourageant la «rue Oudinot» à se pencher prioritairement sur ses revendications statutaires.

Notre droit constitutionnel, longtemps présenté comme dressant un obstacle juridique radical à la prise en compte de telles revendications, au nom de l'« unité » et de l'«indivisibilité » de la République, s'est en réalité avéré plus souple et plus riche que la doctrine n'acceptait de le reconnaître : le législateur pourrait y puiser des solutions institutionnelles peut-être aptes à favoriser un développement plus harmonieux de la Guyane.

Ces solutions devraient naturellement prendre en compte les particularités du pays, notamment eu égard aux autres départements d'outremer.

\section{I. - LA NECESSAIRE PRISE EN COMPTE DES PARTICULARITES GUYANAISES}

1. Sur le plan géographique, tout d'abord, on doit relever l'étendue du territoire et l'impossibilité pour les pouvoirs publics de s'en assurer la maîtrise, la concentration de sa faible population sur la bande côtière, la présence de minuscules communautés dans l'intérieur vivant néanmoins de l'exploitation d'aires étendues, enfin l'extrême perméabilité des frontières, permettant une immigration clandestine constante menant jusqu'à Cayenne des milliers de pauvres hères, brésiliens, haïtiens, et surinamiens, pour l'essentiel.

2. Sur le plan humain, ensuite, la Guyane se caractérise par un extrême morcellement ethnique. Les populations originaires sont constituées d'environ 4500 Amérindiens répartis en six groupes ethniques: Arawaks, Palikours, Wayanas, Galibis, Oyampis et Emerillons. Si certains de ces groupes, vivant toujours dans et de la forêt, comme les Wayanas, sont demeurés très à l'écart du monde moderne, d'autres comme les Galibis vivent sur la côte et 
s'intègrent progressivement aux activités contemporaines. Descendants d'esclaves réfugiés dans la forêt, les Noirs dits «marrons » représentaient environ 7000 personnes avant que leur nombre double du fait de la guerre civile au Surinam ; ils sont répartis en quatre groupes - Saramakas, Djukas, Bonis ou Alukus, Paramakas - vivant essentiellement le long du fleuve Maroni qui n'est en aucune manière, à leurs yeux, une frontière. Amérindiens et Noirs «marrons » continuent à vivre, de manière variable d'un groupe à l'autre, selon leur propre droit coutumier ; et les communautés «francisées », soumises - au moins partiellement - au droit de la République, en ont été, de l'avis de beaucoup d'observateurs, assez profondément perturbées et destructurées.

Les Créoles, issus de multiples métissages, constituent toujours le groupe le plus important (environ 50 000), mais les puissantes immigrations récentes l'ont ramené de $70 \%$ à $35 \%$ environ de la population globale. Quoique tolérante et assimilatrice de par son origine même, cette population vit douloureusement la perte de sa domination démographique, même si elle a pu, jusqu'à présent, conserver à peu près intact son monopole sur la vie politique du pays. Elle souffre en outre de la difficulté à définir une identité créole face à des groupes bien moins nombreux, certes, mais à l'identité beaucoup plus affirmée. La «guyanité », tant chantée ces dernières années par certains leaders créoles, demeure une notion bien floue. Et le complexe d'infériorité que semble éprouver cette population envers les Antillais, et singulièrement les Martiniquais, ne l'aide pas à trouver son équilibre.

La Guyane comporte aussi une importante communauté de métropolitains (entre 15 et 20000 personnes) ne séjournant sur place que quelques années, mais détenant la quasi-totalité des fonctions importantes dans les administrations de l'Etat et au Centre spatial guyanais.

On trouve enfin diverses communautés d'origine étrangère, dont l'importance numérique est inversement proportionnelle au poids dans les activités économiques locales: des Libanais (quelques centaines), très implantés dans le commerce de gros et les affaires; des Chinois (environ 
$2000)$ spécialisés dans le commerce de détail ; des Laotiens d'ethnie Hmong (1500) implantés en 1979 et spécialisés dans le maraîchage ; des Surinamiens réfugiés (environ 7 000), qui souhaitent s'intégrer; enfin des Brésiliens (environ 20 000) et des Haïtiens (environ 25 000) fuyant la misère de leur terre natale pour s'infiltrer en Guyane à travers l'Oyapock pour les premiers, à travers le Maroni, via le Surinam, pour les seconds, qui trouvent à s'employer comme main-d'œuvre à bon marché et recherchent eux aussi l'intégration.

Cette population, qui comporte donc environ $45 \%$ d'étrangers récemment immigrés, se caractérise ainsi par une extraordinaire hétérogénéité socio-culturelle, par d'inévitables tensions inter-ethniques, par une constante mobilité des communautés frontalières, enfin par un niveau moyen de formation extrêmement bas.

3. Sur le plan économique, la Guyane, on l'a dit, ne se trouve pas encore dans les inextricables impasses caractérisant les trois D.O.M. insulaires, et maintes perspectives demeurent envisageables. Toutefois l'un des principaux obstacles au développement du pays réside dans son incapacité à exporter vers les pays de la région, où les coûts de production, du fait des salaires pratiqués, demeurent très inférieurs aux siens. La départementalisation de 1946, concrétisée notamment par un considérable gonflement des transferts de fonds publics et de prestations sociales depuis la métropole, permettant la consommation massive de biens bon marché, importés, issus de la production de masse, a en effet transformé le mode de vie, découragé les activités productives traditionnelles, de faible rentabilité, et constitue un obstacle persistant au démarrage de toute activité productive nouvelle. De surcroît, leur association à la C.E.E. confère aux pays dits «A.C.P.» de la région des privilèges destinés à favoriser leurs exportations, et que ne contrebalancent que de légères sujétions, comme la non-discrimination : c'est ainsi qu'ils peuvent, notamment, exporter librement vers les départements français d'Amérique, partie intégrante de l'Union européenne, tout en ayant le droit de se protéger par des droits de douane, des importations en provenance de ceux-ci. Dès lors, on peut envisager l'hypothèse selon laquelle le passage de la Guyane à un statut de «Pays et Territoire d'Outre-mer» au sens de 
l'annexe IV du traité des Communautés européennes, la faisant sortir du champ d'application du droit intra-communautaire au profit d'un régime d'association proche de celui des Etats A.C.P., serait favorable au développement de ses activités productives; toutefois le simple basculement d'un statut de D.O.M. à un statut de T.O.M. en droit interne n'entraînerait pas ipso facto son inscription sur la liste des P.T.O.M. : il faudrait pour cela une modification de cette liste à l'unanimité des Etats membres de l'Union européenne. Enfin, si les départements français d'Amérique sont longtemps restés extérieurs aux accords internationaux de coopération économique censés favoriser l'industrialisation des pays membres (comme le CARICOM) de même qu'aux «initiatives » prises par Washington dans le même sens (comme le C.B.I.), la France est signataire au titre d'Etat associé de la convention de Carthagène (Colombie), qui donna naissance le 29 juillet 1994 à l'Association des Etats de la Caraïbe: cette nouvelle organisation internationale devrait permettre à la Guyane, notamment, de s'insérer dans cet « espace économique élargi pour le commerce et l'investissement ».

4. Contrairement à une opinion répandue, les règles de droit en vigueur en Guyane se sont toujours distinguées, dans une certaine mesure, de celles applicables dans les trois autres «vieilles colonies», le législateur lui ménageant les particularités juridiques qui paraissaient adaptées à sa situation spécifique. La plus notable d'entre elles fut la création, par un décret du 6 juin 1930, du «Territoire de l'Inini » - transformé par une loi du 14 septembre 1951 en «Arrondissement de l'Inini »-, établissement public territorial destiné à soustraire les populations sylvicoles (Amérindiens et Noirs-marrons) à l'administration du Conseil général, composé d'élus créoles peu soucieux de leurs intérêts, pour les placer sous la «protection » de fonctionnaires de l'Etat.

Le remplacement en 1969 de cette réminiscence de l'«administration directe » coloniale par un arrondissement ordinaire n'entraînera néanmoins pas l'application du droit commun aux populations concernées. En effet Amérindiens et Noirs-marrons n'avaient jusqu'en 1967 aucune existence juridique, ne se considérant pas et n'étant pas considérés comme français, mais comme membres de nations ayant fait alliance avec la France ; fut alors 
entreprise, à l'initiative de M. Vignon, sénateur, et du Conseil général, une opération systématique de «francisation » de ces populations par une série de jugements déclaratifs de naissance suivis de la déclaration à l'état civil de leurs enfants, opérations à laquelle certaines communautés se sont néanmoins soustraites. L'octroi de la citoyenneté devait, dans l'esprit des notables créoles comme de l'Administration, avoir un effet assimilateur par l'application à ces populations de l'ensemble des règles et institutions établies par la loi. Cela s'avéra rapidement impossible, et des aménagements de pur fait, dépourvus de toute base légale, durent être mis en œuvre pour ces citoyens français à la situation si particulière :

- les impôts locaux ne sont pas levés ;

- l'appel sous les drapeaux est limité aux jeunes gens qui en font la demande, et à la condition qu'ils soient francophones ;

- l'obligation de scolarisation n'est pas sanctionnée ;

- l'assistance médicale gratuite est généralisée ;

- un arrêté préfectoral du 3 octobre 1977 soumet, sans aucun fondement légal jusqu'ici, l'accès au tiers sud du territoire à autorisation du préfet, laquelle n'est accordée qu'exceptionnelle-ment; le motif invoqué est le danger que courent les expéditions mal préparées, le but réellement poursuivi étant la protection des communautés de la forêt contre tout envahissement, générateur de graves perturbations ;

- enfin, et surtout, les communautés amérindiennes et noires continuent à vivre plus ou moins largement sous l'empire de leur règles coutumières pour tout ce qui touche à la famille et aux biens, les litiges étant soumis à l'arbitrage des chefs coutumiers; cette situation, caractéristique des peuples autochtones de certains de nos territoires d'outre-mer et de Mayotte, est totalement contraire à la légalité en vigueur dans les départements français, mais les représentants des communautés concernées affirment leur désir de voir la République la légaliser. 
Les particularités du régime juridique applicable en Guyane ne s'arrêtent pas là, et plusieurs autres doivent encore être évoquées :

- la quasi-totalité du territoire fait partie du domaine privé de l'Etat, qui en concède l'utilisation aux particuliers et collectivités ;

- les tribus amérindiennes réclament de longue date que la propriété de leurs zones traditionnelles de parcours pour l'exploitation des ressources de la forêt leur soit reconnue; des délimitations ont été effectuées, mais la signature des arrêtés préfectoraux de concession se heurtent à une vive opposition des milieux créoles ;

- la législation minière demeure particulière (d'origine coloniale), de même que le régime forestier ;

- le préfet, héritier en cela du gouverneur, peut suspendre un maire ou un conseil municipal, alors que cette mesure relève normalement d'un décret ;

- aucune T.V.A. n'est perçue ;

- le produit de l'octroi de mer n'est pas versé exclusivement aux communes, mais aussi, à hauteur de $35 \%$, au département.

5. Aucune réflexion sur une évolution statutaire de la Guyane ne peut, enfin, s'effectuer dans l'ignorance des particularités de la vie politique locale. Dans une société aussi segmentée ethniquement que l'est la société guyanaise, où nulle industrialisation massive n'est venue superposer aux communautés en présence le clivage en classes sociales qui seul correspond aux logiques des mécanismes démocratiques, la transposition de ces mécanismes ne fait illusion que sur l'observateur pressé. Créé en 1956 par Justin Catayee, le Parti socialiste guyanais (P.S.G.) développe avec constance une rhétorique d'inspiration marxiste et tiers-mondiste lui conférant un ascendant considérable sur l'élite créole et une situation de parti dominant auquel il paraît téméraire de s'opposer. S'appuyant sur cette situation de quasimonopole politique, la communauté créole, la plus moderne par sa formation donc la plus apte à s'insérer dans les institutions publiques, a tiré profit du développement de la décentralisation pour accentuer son monopole sur les postes électifs comme sa mainmise sur l'intérieur du territoire, où elle est pourtant très peu présente. Et la transposition en «zone tribale» des 
mécanismes électoraux prévus par la République s'y est traduite par de déplorables manœuvres de corruption et d'achats de votes collectifs au détriment des communautés amérindiennes et de Noirs-marrons, dont les frustrations se sont trouvées aggravées, conduisant leurs représentants à appeler de leurs vœux un «retour» du préfet, apprécié pour la neutralité de son arbitrage, ainsi que l'adoption à leur profit, par la loi, d'un statut préservant leur identité culturelle et leur autonomie sociale.

Quant au rôle de Paris, et de son préfet, dans l'administration des affaires guyanaises, il cristallise toutes les ambiguités et les complexités des sensibilités locales : la communauté créole le vilipende volontiers, réclamant bruyamment plus d'autonomie... mais se réjouit secrètement d'y trouver un commode bouc émissaire ; les communautés tribales, pour leur part, appellent de leurs vœux la «protection» de l'Etat et de ses représentants, mais souhaitent demeurer à la périphérie de la République.

Ainsi résumées, les particularités guyanaises devraient inspirer aux pouvoirs publics quatre préoccupations essentielles dans toute réflexion sur une évolution statutaire du pays :

- mettre un terme à la superposition département/région, non-sens administratif entraîné par la hâte dans laquelle le Gouvernement dût tirer les conséquences de la décision du Conseil constitutionnel du 2 décembre 1982 ;

- approfondir la décentralisation en développant, conformément aux demandes formulées par les élites locales, les transferts de compétences de l'Etat aux organes décentralisés ;

- instaurer des contre-pouvoirs et des mécanismes de responsabili-sation des élus locaux, afin de contenir la propension des élites du P.S.G. au monopole politique et institutionnel ;

- organiser une représentation distincte des «populations tribales », dont la culture et les intérêts, extérieurs aux logiques économiques, administratives et politiques contemporaines, ne pourront pas, autrement, trouver à s'exprimer. 
Une gamme de solutions institutionnelles peut d'emblée être envisagée.

\section{II. - PREMIERE REFORME ENVISAGEABLE : SUBSTITUER A LA REGION UNE COLLECTIVITE A EXECUTIF RESPONSABLE}

La réforme la plus simple pourrait être calquée sur celle adoptée pour la Corse par la loi du 13 mai 1991. Elle consisterait à substituer à la région et à elle seule, le département étant donc conservé - une collectivité territoriale de type particulier fondée sur la disposition de l'article 72 de la Constitution selon laquelle le législateur peut créer toute collectivité territoriale autre que celles (commune, département, et territoire d'outre-mer) désignées dans ce même article 72. S'affranchissant ainsi du principe d'uniformité intra-catégorielle auquel la jurisprudence constitutionnelle a donné une grande rigueur - et ce, même pour les départements et régions d'outre-mer, auxquels l'article 73, interprété de manière très restrictive par le Conseil constitutionnel, ne permet guère de se singulariser - le législateur se trouverait très libre de ses mouvements, comme l'a montré notamment la décision du Conseil constitutionnel du 9 mai 1991 sur le nouveau statut de la Corse.

Il pourrait dès lors transposer en Guyane l'organisation de type quasi parlementaire appliquée à la Corse, elle-même inspirée de techniques juridiques empruntées à la fois au statut de la Polynésie française, à la Constitution de la $\mathrm{V}^{\text {ème }}$ République, et à la Loi fondamentale de la République fédérale allemande. Les originalités essentielles du statut de la Corse sont les suivantes :

- une assemblée très surdimensionnée par rapport à la population de l'île, eu égard aux normes en vigueur pour les Conseils régionaux ;

- un mode de scrutin proportionnel affecté d'un correctif majoritaire : la liste venue en tête (au premier tour une majorité absolue est exigée) reçoit 
trois sièges avant de participer à la répartition des quarante-huit autres sièges ;

- un «Conseil exécutif » distinct du président et du bureau de l'assemblée, et composé d'un président et de six conseillers élus par celle-ci au scrutin de liste majoritaire à trois tours... et qui perdent immédiatement leur siège à l'assemblée ;

- cet exécutif fonctionne, à l'instar d'un gouvernement, de manière largement collégiale, les plus importants des arrêtés de son président devant faire l'objet d'une délibération en son sein, et quatre au moins des six conseillers devant être chargés d'attributions effectives précisées par la loi ;

- ce Conseil exécutif est collectivement responsable de sa gestion devant l'assemblée; en effet une motion de défiance peut être déposée devant celle-ci, revêtue de la signature d'au moins un tiers de ses membres, comportant en premier lieu les motifs pour lesquels la confiance est retirée au Conseil exécutif, puis (technique inspirée de la Loi fondamentale allemande) la liste des candidats aux fonctions de président et de conseillers exécutifs disposés à entrer en fonction au lieu et place de l'équipe désavouée. Le vote de cette motion ne peut avoir lieu qu'après l'écoulement d'un délai de quarante-huit heures après son dépôt. Elle ne sera considérée comme adoptée que si elle a recueilli (règle transposée de l'article 49 de la Constitution française de 1958) les suffrages de la majorité absolue des membres composant l'assemblée. Après son adoption éventuelle, le nouveau Conseil exécutif entre immédiatement en fonction.

Telles sont les originalités principales du statut de la «collectivité territoriale » de Corse. Il s'agit, on le voit, de mettre en place des mécanismes de responsabilité effective de l'équipe exécutive devant l'assemblée imposant aux élus de régler eux-mêmes les désaccords survenant entre les deux organes... alors que l'organisation des collectivités territoriales de droit commun n'offre d'autre issue à ces désaccords qu'une paralysie des 
institutions débouchant sur la dissolution de l'assemblée par décret et de nouvelles élections.

En d'autres termes, les élus locaux se trouvent ainsi dans une situation de plus grande autonomie organique, sans pour autant jouir nécessairement d'attributions plus développées. Et cette situation devrait favoriser la formation d'une culture plus moderne, moins «relationnelle», plus «fonctionnelle », plus « républicaine », dans la gestion de l'intérêt général.

De nombreuses variations seraient tout-à-fait possibles autour de ce schéma, le législateur n'étant pas tenu, on l'a dit, de s'aligner sur l'organisation d'une catégorie existante de collectivités territoriales.

Il s'agirait là en quelque sorte de la réforme minimum, ne portant en rien atteinte au statut départemental. Elle accentuerait la décentralisation sans nécessairement accroître les compétences des autorités élues, et favoriserait une maturation de la culture politique guyanaise sans paraître une «aventure » aux yeux de quiconque.

Bien évidemment, elle ne résoudrait aucunement le non-sens administratif que constitue la superposition de deux collectivités décentralisées sur un même territoire, et ne répondrait pas à la demande formulée en ce sens à Cayenne.

\section{III. - SUPPRIMER LA REGION, EN TRANSFERANT SES COMPETENCES AU DEPARTEMENT}

La revendication d'une collectivité territoriale unique, cohérente avec le souci d'une gestion plus rationnelle des affaires locales, trouverait satisfaction plus aisément par la suppression de la région que par celle du département, collectivité territoriale figurant dans la Constitution et représentant, pour les « vieilles colonies », leur ancrage à la République.

Il serait donc envisageable de supprimer la région et de transférer ses attributions aux organes départementaux. On en reviendrait ainsi à une 
collectivité territoriale unique, conformément aux vœux réitérés des élus guyanais, sans rompre avec le statut départemental, mais en conférant à ce département des compétences plus larges que celles du droit commun, même ultra-marin. Cette réforme serait d'autant plus praticable outre-mer que les régions n'y exercent, et pour cause, aucune fonction de coordination des initiatives des départements.

Néanmoins une telle opération ne pourrait à elle seule répondre aux besoins qui s'expriment en Guyane. En premier lieu il importerait - on l'a dit et on y reviendra - de flanquer le Conseil général d'un organe collégial spécifique représentant les «populations tribales ». En second lieu, il pourrait paraître souhaitable de transposer au niveau des organes de ce département à compétences élargies la technique de l'exécutif collégial et responsable dont la collectivité territoriale de Corse a été dotée.

De telles innovations apportées au statut d'un département, fût-il «d'outre-mer», seraient malheureusement susceptibles de se heurter à la jurisprudence du Conseil constitutionnel. Celui-ci, on le sait, a exprimé, notamment par sa décision du 2 décembre 1982, une conception très rigoureuse du principe d'uniformité interne de nos catégories de collectivités territoriales et des possibilités d' «adaptation» qu'ouvre pour les D.O.M. l'article 73 de la Constitution. La loi adoptée par l'Assemblée nationale en dernière lecture le 23 novembre 1982 superposait outre-mer une région à chaque département, mais les dotait d'organes communs, à l'instar de la ville et du département de Paris. Le «Conseil général et régional » devait être élu au scrutin proportionnel, dans le cadre d'une circonscription unique. Saisi par des parlementaires de l'opposition, le Conseil constitutionnel estima que ce mode de scrutin n'aurait pas permis à cette assemblée de représenter les « composantes territoriales du département » que sont à ses yeux les cantons, donc que cette assemblée n'aurait pas été un Conseil général ; et cette atteinte à l'organisation départementale aurait excédé les simples «mesures d'adaptation» permises pour les D.O.M. par l'article 73 de la Constitution mais aurait revêtu le caractère d'une « organisation particulière », que l'article 
74 réserve aux T.O.M. Donc une telle modification du statut d'un département aurait été contraire à la Constitution.

Cette très importante décision, complétée ultérieurement par d'autres, a ramené à peu de choses les «mesures d'adaptation » permises par l'article 73. Et la doctrine considère qu'aujourd'hui la voie de la collectivité particulière, ouverte, on l'a vu, par la «petite phrase » de l'article 72 et la décision du Conseil constitutionnel du 25 février 1982, offre beaucoup plus de possibilités... en permettant au législateur de s'affranchir de l'exigence d'uniformité de la catégorie des départements.

Quel degré d'innovation la Haute-Juridiction serait-elle aujourd'hui disposée à accepter sur le statut d'un département d'outre-mer ? Nul ne peut le prédire, les critères d'appréciation des Neuf Sages comportant inévitablement un aspect politique, chacun d'eux estimant en conscience l'opportunité des mesures soumises au Conseil...

On peut toutefois envisager que la première des trois innovations envisagées ci-dessus (transférer au département de la Guyane les attributions de la région, qui serait supprimée) devrait pouvoir être acceptée par le Conseil constitutionnel, comme ne dépassant pas les «mesures d'adaptation nécessitées par la situation particulière» (formule de l'article 73) de ce département d'outre-mer; les conséquences notoirement regrettables de sa décision particulièrement restrictive du 2 décembre 1982 pourraient laisser envisager, comme en compensation, une plus grande souplesse de sa part sur ce point.

La seconde innovation suggérée (instituer, à côté du Conseil général, une assemblée représentant les «populations tribales») serait très probablement acceptée si cette assemblée n'avait qu'une fonction consultative, mais le serait beaucoup plus difficilement si le législateur lui conférait - ce qui paraît hautement souhaitable - une compétence délibérante, instaurant ainsi un bicaméralisme départemental probablement trop audacieux aux yeux des Neuf Sages... 
Quant à la troisième innovation proposée (la mise en place d'un exécutif distinct du bureau du Conseil général et que celui-ci pourrait renverser par une motion de défiance), elle apparaîtrait probablement, elle aussi, à la Haute-Juridiction, comme dotant le département de la Guyane d'une « organisation particulière » que la Constitution réserve aux T.O.M.

Le maintien du département ne permettrait donc pas à la loi d'aller très loin dans la recherche d'institutions « adaptées ».

\section{IV. - CREER UNE COLLECTIVITE TERRITORIALE UNIQUE ET SPECIFIQUE, CONSERVANT LE REGIME LEGISLATIF DE L'ARTICLE 73}

La formule utilisée autrefois pour l'Algérie (collectivité territoriale originale regroupant plusieurs départements) puis plus récemment, et avec l'aval explicite du Conseil constitutionnel, pour Paris, Mayotte, Saint-Pierreet-Miquelon, et la Corse, donne au législateur les coudées très franches pour élaborer les institutions de son choix. La collectivité territoriale spécifique, extérieure aux catégories existantes, n'est pas tenue de s'aligner (avec simplement de légères variantes) sur le schéma général d'une catégorie. Le Conseil constitutionnel estime en effet que le principe d'égalité, qui impose à ses yeux une uniformité intra-catégorielle assez poussée, n'est dans ces conditions pas menacé, la collectivité territoriale en question étant seule de son espèce.

Remplacer, en Guyane, la région et le département par une collectivité territoriale unique «sui generis » permettrait donc au législateur de mettre sur pied une organisation originale susceptible de faire face aux différents besoins qui s'expriment dans le pays. Un exécutif collégial distinct du bureau de l'assemblée et pouvant être renversé par celle-ci, s'inspirant donc d'un gouvernement de régime parlementaire ; une assemblée élue selon un mode de scrutin original, qui pourrait n'être ni celui des conseils généraux ni celui des conseils régionaux, apte par exemple à assurer une sur-représentation des 
groupes ethniques les moins nombreux; des compétences importantes susceptibles de dépasser, par de nouveaux transferts de la part de l'Etat, celles, cumulées, de la région et du département actuels; la création d'un organe collégial représentant les «populations tribales", même investi d'une fonction délibérante le situant, comme en «bicaméralisme égalitaire », sur le même plan que la première assemblée... tels pourraient être quelques-uns des caractères des nouvelles institutions guyanaises, le Conseil constitutionnel ne pouvant y trouver à redire que si se trouvait supprimé l'indispensable « contrôle administratif » exercé par un « délégué du Gouvernement » (article 72) sur lequel repose, en dernière analyse, le caractère unitaire de l'Etat.

Afin de maintenir un régime d'application de plein droit (avec d'éventuelles adaptations) des lois et décrets, la loi établissant la nouvelle collectivité territoriale devrait spécifier que celle-ci demeure placée sous l'empire de l'article 73 de la Constitution. Ainsi le développement de l'autonomie locale ne se traduirait pas par une rupture avec l'ordre juridique de droit commun, ni par le sentiment d'une « sortie de la République ». Ainsi, en outre, la nouvelle collectivité territoriale prendrait-elle le relais des deux collectivités actuelles au sein de la Communauté européenne, la jurisprudence «Hansen » de la Cour de Justice des Communautés européennes (1978) selon laquelle les D.O.M. relèvent en principe du droit communautaire commun - lui demeurant applicable.

Il faut toutefois préciser que la sortie du statut départemental pourrait peut-être se heurter à des obstacles d'ordre constitutionnel. En premier lieu, l'existence des départements - contrairement à celle des régions - est prévue par la Constitution, même si celle-ci n'en fournit pas la liste ni n'indique les territoires qu'ils recouvrent. Et la sortie de Saint-Pierre-et-Miquelon du statut départemental, effectuée en 1985, ne pourrait servir de précédent, la loi érigeant l'archipel en collectivité territoriale particulière n'ayant pas été portée devant le Conseil constitutionnel. En second lieu, les Neuf Sages pourraient estimer que la Constitution scinde le territoire national en deux parties, et deux seulement, les territoires d'outre-mer, placés sous le régime de la « spécialité législative », et les départements, sous l'empire du droit commun : 
les premiers seuls seraient susceptibles de changement de statut à l'intérieur de la République, les seconds en étant écartés sous peine de porter atteinte à l'« indivisibilité » de l'Etat.

Rien ne permet d'affirmer que le Conseil constitutionnel s'opposerait à une sortie de la catégorie des départements mais, l'ordre public n'étant pas gravement perturbé, en Guyane, par les partisans d'une telle réforme, on peut craindre que la Haute-Juridiction ne soit tentée de faire prévaloir une vision conservatrice de la structure de la République.

\section{V. - CREER UNE COLLECTIVITE TERRITORIALE UNIQUE ET SPECIFIQUE PLACEE SOUS UN REGIME DE SPECIALITE LEGISLATIVE PARTIELLE}

Rassurant pour les tenants de la politique d'intégration (à la Nation française comme à l'Union européenne) le statut proposé au point précédent ne répondrait par contre que fort timidement - à la mesure d'éventuels transferts de compétences supplémentaires - aux demandes de ceux qui, en Guyane, réclament que les règles de droit appliquées localement soient édictées localement afin d'être mieux adaptées aux problèmes du pays.

Il serait alors possible au législateur, s'inspirant du statut de l'Algérie (loi du 20 septembre 1947), d'établir au profit de la nouvelle collectivité territoriale une «spécialité législative» partielle. Un certain nombre de domaines demeureraient placés sous l'empire des lois et décrets ordinaires, éventuellement «adaptés », comme pour les départements d'outre-mer, mais d'autres domaines ne pourraient être réglés que par une manifestation de volonté expresse du législateur et du pouvoir réglementaire, soit insérée dans un texte général, soit prenant la forme d'une modification de ce texte général, soit se traduisant par l'adoption de lois et décrets spécifiques, à l'instar du régime des territoires d'outre-mer. Un certain nombre de matières de cette seconde catégorie pourraient en outre - comme pour l'Algérie du statut de 1947 - être transférées du Gouvernement et même du législateur aux autorités 
locales. Alors que les territoires d'outre-mer et Mayotte relèvent totalement de ce régime législatif spécial, la nouvelle collectivité territoriale guyanaise pourrait n'en relever qu'en partie, et être un D.O.M. dans certaines matières et un T.O.M. dans d'autres. Ainsi pourraient se trouver conciliées les demandes contradictoires d'intégration à la République et de prise en compte des particularismes.

On ajoutera qu'une technique juridique des plus classiques permettrait de mettre l'accent soit sur l'application du droit commun avec adaptations (D.O.M.) soit sur l'élaboration de règles spécifiques (T.O.M.) : la loi pourrait en effet soit donner la liste des matières relevant de textes spécifiques, conservant tout le reste sous l'empire du droit commun éventuellement adapté (accent sur l'intégration), soit énoncer, au contraire, la liste des domaines demeurant par principe régis par les textes généraux, remettant tout le reste à des manifestations de volonté expresses du législateur ou du pouvoir réglementaire (accent sur la spécificité de traitement). Le choix pour l'un ou l'autre de ces procédés de répartition serait en outre susceptible d'influer sur l'attitude des institutions communautaires envers la nouvelle collectivité : Paris pourrait demander aux Etats membres et à la Conférence intergouvernementale l'inscription de la Guyane dans la liste des P.T.O.M. si la nouvelle collectivité territoriale relevait en principe - en droit interne - de textes spécifiques.

\section{VI. - CREER UN TERRITOIRE D'OUTRE-MER}

La Constitution ne comportant ni définition ni liste des territoires d'outre-mer, la loi - une loi organique depuis la réforme constitutionnelle du 25 juin 1992 - aurait toute latitude pour faire passer la Guyane dans cette catégorie de collectivités territoriales, réserve faite de ce qui a été dit plus haut sur les éventuelles objections que le Conseil constitutionnel serait susceptible de formuler face à une sortie du statut départemental. 
La création d'un territoire d'outre-mer aurait les effets juridiques suivants :

- conférer la plus grande liberté au législateur pour doter la Guyane de l'organisation de son choix, sur un large registre s'étendant d'une simple administration déconcentrée par un représentant de l'Etat, d'une part, jusqu'à des organes directement inspirés de ceux d'un Etat, d'autre part, jouissant de compétences considérables, en d'autres termes jusqu'à l'autonomie interne; l'archipel de Wallis-et-Futuna présente un cas de T.O.M. extrêmement peu décentralisé, essentiellement placé sous l'autorité de l'Administrateur supérieur, alors que le Territoire français des Afars et des Issas a naguère, en sens inverse, fourni l'exemple d'un T.O.M. bénéficiant d'une considérable autonomie évoquant la situation d'un Etat fédéré ;

- placer la Guyane sous le régime exclusif de la «spécialité législative » : hormis dans quelques domaines précis touchant aux questions de souveraineté, les lois et décrets adoptés à Paris ne lui seraient pas applicables, sauf manifestations expresses de volonté en sens inverse du législateur ou du pouvoir réglementaire, généralement assorties d'adaptations ; mais, dans la plupart des domaines, un T.O.M. est régi par des textes spécifiquement rédigés pour s'adapter à sa situation particulière ;

- entraîner éventuellement la sortie de la Guyane du champ d'application du droit intra-communautaire et son inscription sur la liste des «Pays et Territoires d'Outre-mer», liés à la Communauté européenne par un régime d' "association » jusqu'à présent largement inspiré de celui des pays dits «A.C.P.» (Afrique, Caraïbes, Pacifique); les conséquences en pourraient être résumées ainsi: maintien du libre accès au marché communautaire mais droit de se protéger contre les importations en provenance de l'Union, fin du bénéfice des financements par les «Fonds structurels » que sont le Fonds européen d'Orientation et de Garantie agricole (F.E.O.G.A.), le Fonds européen de Développement régional (F.E.D.E.R.) et le Fonds social européen (F.S.E.), mais accès aux 
financements du Fonds européen de Développement (F.E.D.) et du Système de Stabilisation des Recettes d'Exportation (STABEX), dont les apports seraient très inférieurs aux précédents.

On le voit, placer la Guyane sous le régime de l'article 74 de la Constitution équivaudrait à revenir sur la politique d'intégration (à la France et peut-être aussi à la Communauté européenne) menée depuis 1946 et renforcée par les mesures sociales prises au début des années 90 en application du rapport Ripert, mais permettrait une meilleure prise en compte des problèmes locaux et un considérable approfondissement de la décentralisation, voire la mise en place de l'autonomie. Sur le plan économique, l'éventuel basculement de la Guyane dans la catégorie des P.T.O.M. entraînerait une considérable diminution des financements communautaires, mais le pays pourrait en contrepartie se protéger des importations bon marché en provenance du territoire communautaire, ce qu'il ne peut pas faire actuellement en principe. Le développement des activités productives en serait probablement stimulé, mais le pouvoir d'achat moyen abaissé. L'allégement de la dépendance, aux effets certainement positifs dans maints domaines, se paierait sans doute, en termes de niveau de vie, d'un coût estimé excessif.

\section{VII. - ORGANISER UNE REPRESENTATION DISTINCTE DES « POPULATIONS TRIBALES »}

Quel que soit le statut proposé à la Guyane, il semble indispensable d'y intégrer une représentation spécifique des communautés amérindiennes ou de « Noirs-marrons ».

On l'a évoqué, ces populations ont, au moins en partie, été intégrées sans leur réel consentement à un système administratif dont la logique leur est totalement étrangère, qui exerce sur elles de puissants effets destructurants, qui échappe à leur contrôle mais accentue, en sens inverse, la domination qu'exerce sur elles la communauté créole. Si, pour diverses raisons, il paraît 
impossible de revenir en arrière - par exemple, supprimer les allocations familiales serait inconcevable, même si leurs effets semblent avoir été globalement négatifs - il importe à l'inverse d'institutionnaliser l'expression par ces communautés de leurs intérêts, et de leur conférer les moyens d'une réelle participation à l'administration de la Guyane.

Divers types d'organes, déjà évoqués, pourraient remplir un tel rôle. Le moins audacieux serait un conseil consultatif coutumier placé, à l'image de celui qui fonctionne actuellement en Nouvelle-Calédonie, auprès des organes délibérant et exécutif. Ses membres seraient des délégués des diverses communautés, désignés par chacune d'elles selon ses propres usages.

Plus audacieuse serait la constitution d'une seconde assemblée délibérante représentant non plus les individus mais les groupes ethniques eux-mêmes, indépendamment de leur poids démographique, selon le principe paritaire qui permet au Sénat des Etats-Unis de représenter sur un pied d'égalité tous les Etats de l'Union, quels que soient leur population et leur poids économique, en leur attribuant à chacun deux sièges. Toutefois le rattachement à telle ou telle communauté, incompatible avec les principes fondateurs de notre République, soulèverait en outre une multitude de cas délicats. Peut-être la solution pourrait-elle être recherchée du côté d'une représentation des communes qui, au nombre de vingt-et-une, semblent pour beaucoup d'elles correspondre majoritairement à une communauté ethnique ? Quelques communes nouvelles pourraient auparavant être créées pour conférer un support territorial à des groupes encore très mobiles.

A côté d'une telle représentation, la création à Cayenne d'un service administratif chargé de suivre exclusivement les questions touchant aux Amérindiens et Noirs-marrons paraît indispensable, à condition qu'il soit placé sous la direction de personnes réellement qualifiées et non pas de hautsfonctionnaires de passage.

On peut enfin évoquer une structure institutionnelle originale mise en place en Nouvelle-Calédonie sous l'impulsion de M. Pisani, lequel avait pris conscience de ce que «l'autonomie... (avait) servi de fondement à la 
domination d'une communauté dépositaire du pouvoir politique et du pouvoir économique à la fois », et que l'on peut qualifier de «fédéralisme sous tutelle ». Les organes de la collectivité territoriale de Guyane seraient ramenés à peu de choses, l'essentiel de leurs compétences étant transmis pour partie à deux collectivités territoriales de niveau inférieur, pour partie au représentant du Gouvernement. Les deux collectivités territoriales évoquées seraient l'une, essentiellement côtière, placée sous le régime de l'article 73 de la Constitution (celui des D.O.M.), l'autre, correspondant surtout à l'intérieur (mais les tribus côtières pourraient lui être rattachées), placée sous le régime de l'article 74 (celui des T.O.M.). Auprès du représentant du Gouvernement siégerait une assemblée composée de la réunion des assemblées des deux collectivités composantes. Ainsi pourraient se trouver assurés aussi bien l'administration de ces deux univers selon des logiques distinctes, que l'arbitrage entre eux par une autorité de l'Etat.

Plus délicat encore que le problème de la représentation spécifique des «populations tribales » est celui de la légalisation de leur droit coutumier. La situation actuelle, en effet, est de pur fait, et totalement illégale voire anticonstitutionnelle. La jurisprudence du Conseil constitutionnel s'avère fort claire: les grands principes d'indivisibilité de la République et d'égalité imposent l'application des règles du droit commun à l'ensemble des citoyens résidant dans les départements. Les «mesures d'adaptation » prévues pour les D.O.M. par l'article 73 ont été, on l'a vu, interprétées de manière extrêmement restrictive, la Haute-Juridiction ne leur donnant son aval que si elles lui paraissent véritablement «nécessitées par (une) situation particulière ». La doctrine en a même conclu que cet article, ainsi interprété, s'avère non seulement inutile car n'apportant rien au droit qu'ont toujours le législateur et le pouvoir réglementaire de prévoir des règles particulières pour des catégories particulières de situations, mais très restrictif, en soumettant ces «mesures d'adaptation» à l'exigence exceptionnellement stricte d'une « situation particulière ». Autrement dit, cet article 73 jouerait aujourd'hui l'effet inverse de celui pour lequel il semble avoir été conçu... 
En revanche, les Neuf Sages ont été amenés à indiquer que l'« organisation particulière » prévue pour les T.O.M. par l'article 74 recouvre non seulement la structure des institutions administratives locales (sens évident) mais aussi la nature même des règles de droit applicables à la population du territoire : ces règles, comme les institutions administratives, doivent (et non seulement «peuvent») être «particulières », c'est-à-dire issues d'une manifestation de volonté du législateur ou du pouvoir réglementaire, qui soit spécifique à chaque territoire. Et c'est dans ce cadre, et dans ce cadre seulement, que la loi peut prévoir que la population autochtone d'un territoire conservera son droit coutumier pour tout ce qui a trait au «statut personnel» (famille et biens, surtout), tout en ouvrant à chaque individu la possibilité d'y renoncer pour passer sous l'empire du droit écrit, édicté par la Loi et le Règlement.

En pratique d'ailleurs, seuls les territoires placés sous le régime de la «spécialité législative» de manière expresse présentent des populations légalement régies par un droit coutumier et des juridictions coutumières : il s'agit des Territoires d'Outre-mer et de la collectivité territoriale particulière de Mayotte. Leurs statuts prévoient en effet que les lois et décrets ne s'y appliquent «que sur mention expresse».

Les seuls départements où une telle situation fut reconnue furent les départements algériens. Mais ils se trouvaient précisément à mi-chemin entre le régime des D.O.M. et celui des T.O.M. car, on l'a vu, ils relevaient pour partie des lois et décrets généraux, pour partie de textes spécifiques.

En d'autres termes, l'état actuel de notre droit constitutionnel semble rendre inconciliables le maintien du statut départemental et la légalisation du droit coutumier des «populations tribales ». Cette dernière opération exigerait le passage soit à un statut de territoire d'outre-mer, soit à un statut de collectivité territoriale particulière à laquelle lois et décrets ne s'appliqueraient que sur la volonté explicitement formulée du législateur ou du pouvoir réglementaire, comme à Mayotte. Mais cette situation, on l'a suggéré plus haut, pourrait ne concerner qu'une partie du territoire de la Guyane. 
L'extrême complexité de la situation guyanaise constitue de toute évidence un défi à la rationalité républicaine et jacobine. Mais il devrait être possible de puiser dans un arsenal de techniques juridiques plus riche qu'on ne le pense généralement un cadre institutionnel permettant une évolution harmonieuse de ce pays.

\section{SOURCES}

\section{ENTRETIENS}

Dujus A., Receveur des Postes à Kourou de 1983 à 1991.

GACHET J.-P., INRA « Systèmes agraires et développement », Kourou.

GREnAND P. et F., Ethnologues (Amérindiens).

Jolivet M.-J., Ethnologue (créoles de Guyane).

LACROIX J.-P., ancien Préfet de la Guyane.

VERNON D., Ethnologue (Noirs- marrons ).

VIVIER M., INRA « Systèmes agraires et développement », Kourou.

\section{PUBLICATIONS}

ARnoux I., «Les Amérindiens dans le département de la Guyane : problèmes juridiques et politiques », Revue de Droit public, 1996, n 6, p. 1615.

CENADDOM, Les Dossiers de l'Outre-mer, $\mathrm{n}^{\circ}$ spécial Guyane, 1985.

DOUENCE J.-Cl., «Les Départements d'outre-mer », in Jurisclasseur des Collectivités locales, p. 1900.1 et suivantes.

DouENCE J.-Cl., « Le Droit de l'Environnement dans les départements d'outremer », Rapport à la Société française de Droit de l'Environnement, Université de Pau et des Pays de l'Adour, 1991.

«Des Droits et des Coutumes de Guyane », Compte-rendu d'une journée d'étude organisée par l'antenne Antilles-Guyane de l'Ecole nationale de la Magistrature, in France-Guyane, 16 décembre 1991. 
Revue ETHNIES, «La Question amérindienne en Guyane française », Numéro spécial 1985, réédition 1988.

GRENAND P. et F., «Les Amérindiens, des peuples pour la Guyane de demain », Centre ORSTOM de Cayenne, 1990.

GrEnAND P. et F., «L'impact sociologique des prestations familiales sur les Amérindiens de l'Oyapock », Rapport au ministère des D.O.M./T.O.M., 1985.

GRENAND F., «Famille et corps social : cohérence interne et conflit avec l'extérieur », Communication aux journées d'étude «Familles en Guyane », Cayenne, 30-31 janvier 1992.

GrENAND F., « Problèmes spécifiques aux populations amérindiennes du HautMaroni », Compte-rendu d'un séminaire tenu en pays wayana les 24 et 25 octobre 1990.

GRENAND P. et F., « Note au préfet de la Guyane sur l'impact du R.M.I. sur les Amérindiens de la commune de Camopi », Septembre 1990.

Grenand F., «Enseignement et cultures amérindiennes », in Pour un nouvel enseignement en pays amérindien, Centre ORSTOM de Cayenne, 1990.

INSEE, service régional, « Tableaux économiques de la Guyane », 1995.

Jones B. et Stephenson E., «Politique et société à la Guyane », in Les Antilles-Guyane au rendez-vous de l'Europe, sous la direction de BURTON R. et RENO F., Economica, 1994, p. 101.

JOLIVET M.-J., «Entre autochtones et immigrants : diversité et logiques des positions créoles guyanaise », Communication au $\mathrm{VI}^{\mathrm{ème}}$ colloque international des études créoles, Cayenne, 29 septembre-6 octobre 1989.

MiCHALON Th., «La République française, une fédération qui s’ignore ?», Revue de droit public, 1982, p. 623.

MiCHALON Th., «La corse entre décentralisation et autonomie : vers la fin des catégories ? », Revue française de droit administratif, 1991, n 5, p. 720.

Miclo F., «Institutions publiques des départements d'outre-mer (textes et documents) », Centre universitaire de la Réunion, 1981.

Ministère des Départements et Territoires d'Outre-mer, « La Lettre de l'Outremer », 1989-91.

Mouvement guyanais de décolonisation, Quelques textes essentiels, Cayenne, 1977. 
ORSTOM, Centre de Cayenne, «Dynamique de la population et migrations en Guyane », 1988.

Résolutions du VII ${ }^{\mathrm{ème}}$ congrès du Parti socialiste guyanais (P.S.G.), novembre 1991.

RIPERT (Commission), «L'égalité sociale et le développement économique dans les D.O.M », Rapport au Ministre des D.O.M./T.O.M., 1990.

Rouland N., «Etre Amérindien en Guyane française : de quel droit ? », Revue française de Droit constitutionnel, $\mathrm{n}^{\circ} 27,1996, \mathrm{p} .493-522$.

SABLE V., «La politique de coopération régionale entre les D.O.M./T.O.M. et les Etats A.C.P. », Rapport au Premier Ministre, 1986.

Taubira-Delanon Ch., «Mon peuple «Quonvwé »», in Reno F. (dir.), Identité et politique. De la Caraïbe et de l'Europe multiculturelles, Economica, 1995, p. 121.

TOulEmonde-Niaussat M., «Histoire d'«ethnies »: éléments pour une analyse des mouvements de population sur le Maroni », 1989.

VERNON D., «Eléments d'identité du peuple Ndjucka », 1989.

VIMON J., «Assimilation et dédoublement des ordres normatifs : le cas des Amérindiens de Guyane française », in Constant F. et Daniel J. (dir.), 1946-1996 : Cinquante ans de départementalisation outre-mer, L'Harmattan, 1997, p. 433.

Vissac B., Gachet J.-P., Dedieu B., Vivier M. et Matheron G., « Maîtrise collective du «Plan vert » et conséquences », INRA, 1991.

VIVIER M., «Développer l'élevage en Guyane française : la tentation technocratique », (Le Plan vert 1975-1986).

\section{OUVRAGES}

BONFAIT N., La question amérindienne en Guyane française: aspects administratifs, juridiques et politiques, Mémoire de D.E.A., Faculté de droit et d'économie de la Martinique, 1991, 125 p.

Budoc R.-L., Antilles-Guyane: quel développement à l'aube de 1992 ?, Publisud, 1990.

CASTOR E., 1981-1985 : La Gauche au pouvoir, pour la Guyane : l'Espoir, L'Harmattan, 1986. 
CHERubini B., Cayenne, ville créole et polyethnique, Karthala-CENADDOM, 1988.

DuPONT L., Les départements français d'Amérique, L'Harmattan, 1988.

JACQUEMART S., La question départementale outre-mer, P.U.F., collection G.R.A.L., 1983 .

Jos E. et PERrot D. (dir.), L'Outre-mer et l'Europe communautaire, Economica, 1994.

Larrieu J. et Martres J.-P. (dir.), Coutumes et Droit en Guyane, Economica, 1993.

LuChaIRe F., Manuel de Droit d'Outre-mer, Sirey, 1949.

LuChaIRE F., Droit d'Outre-mer et de la Coopération, P.U.F., 1966.

LUCHAIRE F., Le juge constitutionnel et le régime législatif des territoires d'outrre-mer, R.D.P., 1994, p. 1621 s.

Michalon Th., La décentralisation: les régimes d'administration locale, Syros, 1988.

Miclo F., Le régime législatif des départements d'outre-mer et l'unité de la République, Economica, 1982.

Mouren-Lascaux P., La Guyane, Karthala, 1990.

Othily G., La Guyane, un avenir, une ambition, Ed. Caribéennes, 1989.

Thierry Michalon

Maître de conférences

Membres du CRPLC

Université des Antilles et de la Guyane 